ANALYSIS

\title{
The true cost of antimicrobial resistance
}

\author{
Richard Smith and Joanna Coast argue that current estimates of the cost of antibiotic resistance \\ are misleading and may result in inadequate investment in tackling the problem
}

\author{
Richard Smith professor of health system economics ${ }^{1}$, Joanna Coast professor of health economics ${ }^{2}$ \\ 'London School of Hygiene and Tropical Medicine, London WC1H 9SH, UK; ${ }^{2}$ School of Health and Population Sciences, University of Birmingham. \\ Birmingham, UK
}

\begin{abstract}
Almost as soon as antibiotics were discovered, we knew that bacteria were able to develop resistance against them. ${ }^{1}$ This is not necessarily a problem, as long as there are other antimicrobials to take their place. During the latter half of the 20th century this was the predominant situation, but no longer. ${ }^{2}$ A rapid decrease in the number of new drugs approved and numerous withdrawals on quality and safety grounds have left the well dry, and it is clear that "the existing classes of antibiotics are probably the best we will ever have."

In light of this, there have been efforts to support interventions that encourage more conservative and appropriate use of antibiotics in an attempt to halt or slow the progress of resistance. ${ }^{4}$ However, this action is often too little and may be too late.

Given that the dangers of resistance are widely acknowledged, why isn't more being done? One reason is that antibiotic resistance has fallen victim to evidence based policy making, which prioritises health problems by economic burden and cost effectiveness of interventions. ${ }^{5}$ Health economists have been unable to show that antibiotic resistance costs enough to be a health priority.
\end{abstract}

\section{Limitations of health economic research}

Ten years ago we published a systematic review on the economics of resistance. ${ }^{6}$ We asked two questions: what is the cost of resistance and what is the cost effectiveness of interventions to reduce it? The lack of research meant we could investigate only the second question. ${ }^{7}$ And even here we concluded that the evidence for the cost effectiveness of interventions for resistance was poor.

We have just performed a rapid review at the Department of Health's request to take into account newer information on the first question. The box summarises our methods and further details are available in the full report. ${ }^{8}$ Estimates of additional cost varied from less than $\$ 5(£ 3 ; € 4)$ to more than $\$ 55000$ per patient episode. This might be explained by type of resistance and how productivity losses are dealt with.
Although there was little intrinsically wrong with these papers, we became concerned that their estimates of the actual economic impact may not be accurate because the research used to produce these estimates is limited in its scope. For example, economic estimates are based on the incremental costs and focus on a specific infectious disease or set of diseases: estimates are based on the cost of extra treatment of a resistant infection compared with susceptible infection, such as costs of additional investigations, more expensive drugs, side effects from extra treatments, longer hospital stay, and greater mortality. ${ }^{9}$ Some may also include costs associated with surveillance and activities associated with trying to control resistance. Most studies were from the United States and based in hospitals and included the costs related to additional hospital stay and treatment but not early mortality.

None of the studies considered the bigger picture- a world in which there are no effective antibiotics for situations where they are currently used routinely, such as in hip replacement or cancer patients. Our concern is that today's limited estimates will be used to project future costs. Will the current worst case scenario place antibiotics high enough up on the health agenda to ensure adequate action?

\section{Current worst case scenario is still an underestimate}

We took the study that found the highest cost of antimicrobial resistance, of $\$ 55 \mathrm{bn}(\$ 20 \mathrm{bn}$ in health service costs and $\$ 35 \mathrm{bn}$ in lost productivity) per year overall to the US, and compared it with economic burden figures for other health problems in the US. These burden figures are taken from a variety of studies, and the dates range considerably, but it is clear that resistance rates fairly low down (table $\downarrow$ ).

However, the costs of resistance could be much higher than these estimates suggest. As an example we estimated the consequences of having no antibiotics for patients having a total hip replacement. Because antibiotics have been used as 


\section{Box 1 Methods used in the review ${ }^{8}$}

We searched for the combinations of terms relating to resistance, antimicrobial and costs. Some papers did not refer to antimicrobial resistance generally but only to particular drugs or micro-organisms. We extended the search terms to focus on meticillin resistant Staphylococcus aureus and vancomycin resistant enterococci, two of the most studied and potentially serious current resistant infections. We included English language studies (empirical or modelling) with data on costs associated with resistance, since 2000. For empirical studies, we selected studies that included a control group with a susceptible infection because the aim was to focus on costs of resistance, rather than costs of infection.

Data extracted included: study design; sample size/modelling approach; relevant micro-organism and drug; cost perspective, year, currency, time frame, discounting approach; resource use included; valuation methods; summary of results.

We identified 192 possible papers, from which 24 were eventually included in our analyses.

prophylaxis and treatment for hospital acquired infection since hip replacements were first performed we looked at information relating to limb amputation as a proxy for what infection rates might have been with and without antimicrobials.

The figure $\Downarrow$ shows the care pathway for patients requiring hip replacement. Currently, prophylaxis is standard practice, and infection rates are about $0.5-2 \%$, so most patients recover without infection, and those who get an infection have it successfully treated. We estimate that without antimicrobials, the rate of postoperative infection is $40-50 \%$ and about $30 \%$ of those with an infection will die. ${ }^{\text {w14-w17 }}$ Thus, removal of antibiotics would increase postoperative infection by $1-50 \%$ and deaths by $0-30 \%$. Of course, at such rates it is likely that the rates of hip replacement would fall, which would increase the burden of morbidity from hip pain.

We recognise that this is a simplistic analysis, with many of the data obtained from literature relating to amputation as a proxy for hip replacement. However, we use it as an example to illustrate and provoke, to emphasise the point that infection rates and their consequences in terms of health service costs and human health may be unimaginable. As we struggle to imagine the clinical consequences, it is easy to see why the economic burden is at present inestimable.

\section{A world without antibiotics}

Modern healthcare was built over the past century on the basis that infections can be prevented or treated using antimicrobials (exemplified by the US surgeon general famously proclaiming in 1968 that "the war against diseases has been won"). ${ }^{10}$

Resistance is said to present a risk that we will fall back into the pre-antibiotic era. ${ }^{11}$ However, this is perhaps optimistic. Our health system is now designed to treat more chronic conditions. Healthcare has become increasingly technological and invasive, improving mortality and morbidity significantly, and antimicrobials have become integrated in many aspects of such care. For example, antimicrobials are given as standard to prevent iatrogenic infection in surgical care, ${ }^{11}$ to women delivering by caesarean section, ${ }^{12}$ and to those having cancer treatment. ${ }^{13}$ From cradle to grave, antimicrobials have become pivotal in safeguarding the overall health of human societies. When viewed in this broader way, the costs of resistance are not limited to those associated with additional treatment for a primary infection , such as a "strep throat." Rather, they must encompass the costs that might relate to the loss of modern healthcare. In the same way that health systems need adequate and effective health workers to function, they also require effective antimicrobials. Resistance is not just an infectious disease issue; it is a surgical issue, a cancer issue, a health system issue.

To calculate the true economic burden of resistance we therefore have to consider the burden associated with not having any effective antimicrobial drugs. And, as witnessed when there are outbreaks of hospital acquired infection, the system can very quickly come to a standstill. ${ }^{14}$ In the future we may need to rethink how the health system is developed-for instance, redesigning many facilities or reintroducing sanatoriums if effective antibiotic treatments are no longer available.

\section{Planning for an uncertain future}

Although we now have more empirical information on the economic burden of resistance than was available a decade ago, it is unlikely to help us plan for the future. Even the highest current estimates of the costs of resistance provide false reassurance and this may mean that inadequate attention and resources are devoted to resolving the problem. Our illustrative example for hip replacement without antibiotics shows how difficult it is to forecast the likely economic burden of resistance. And we have explained how resistance has the potential to undermine modern health systems.

Full health systems analyses seem a more appropriate means to assess the potential impact of resistance and evaluate measures to stem it. Although this approach is complex, understanding the threat to the health system overall, not just for specific diseases, could be the single most important step in better understanding the economic burden that resistance presents. Analysing outbreaks of resistant infections and the cost of disruption to the heathcare system may help produce future estimates. $^{15}$

A change in culture and action is needed to plan for a future with more antibiotic resistance. Considerable inertia remains regarding radical change in our stewardship of antimicrobials, precisely because there is a focus on current economic burden. The same is true with climate change and, to a degree, the financial system. A major driver of this inertia is likely to be collective uncertainty about the clinical and financial implications of increasing resistance. But there is a tried and trusted way to deal with such uncertainty-insurance. Waiting for the burden to become substantial before taking action may mean waiting until it is too late. Rather than see expenditure on antimicrobial policies as a cost, we should think of it as an insurance policy against a catastrophe; albeit one which we hope will never happen. ${ }^{9}$

We thank Callum Hodge, Joseph Griffin, and Daniel Haynes for help with literature searching and Anthony So, Ursula Wells, Claire Boville, Sally Wellsteed, Ross Leach, Peter Bennett, John Henderson, David Cohen, Miranda Mugford, and Huseyin Naci for their comments. This report is independent research commissioned and funded by the Department of Health Policy Research Programme (Economic burden of antimicrobial resistance: a rapid paper, 0410035). The views expressed in this publication are those of the authors and not necessarily those of the Department of Health.

Contributors and sources: Both authors have been health economists for over 20 years and have worked together on aspects related to antimicrobial resistance for more than 15 years. This article is a culmination of experiences over that time, and is based particularly on 


\section{Key messages}

An increase in resistant organisms coupled with a big fall in the number of new antimicrobial drugs suggests an apocalyptic scenario may be looming

Current estimates suggest antimicrobial resistance has a low economic impact

Such estimates do not take into account that antimicrobials are integral to modern healthcare

We may not ever be able to make an accurate forecast of the costs

We should view greater investment in antibiotic resistance as an insurance policy

a rapid review, and interaction with colleagues who commissioned this review at the Department of Health. Both authors contributed equally to the writing of the paper. RS is guarantor.

Competing interests: We have read and understood the BMJ Group policy on declaration of interests and have no relevant interests to declare.

Provenance and peer review: Not commissioned; externally peer reviewed.

1 Courvalin P. Predictable and unpredictable evolution of antibiotic resistance. $J$ Intern Med 2008;264:4-16.

2 So AD, Ruiz-Esparza Q, Gupta N, Cars O. 3Rs for innovating novel antibiotics: sharing resources, risks, and rewards. BMJ 2012:344:e1782.

3 Cormican M, Vellinga A. Existing classes of antibiotics are probably the best we will ever have. BMJ 2012;344:e3369.

4 World Health Organization. The evolving threat of antimicrobial resistance: options for action. WHO, 2012.

5 Coast J, Smith RD, Millar MR. Disentangling value: assessing the benefits of containing antimicrobial resistance. In: Roberts $\mathrm{J}$, ed. The economics of infectious disease. Oxford University Press, 2006:201-14

6 Smith RD, Coast J, Millar MR, Wilton P, Karcher A-M. Interventions against anti-microbial resistance: a review of the literature and exploration of modelling cost-effectiveness. WHO, 2001
7 Wilton P, Smith RD, Coast J, Millar M. Strategies to contain the emergence of antimicrobial resistance: a systematic review of effectiveness and cost-effectiveness. $J$ Health Serv Res Policy 2002;7:111-7.

8 Smith R, Coast J. The economic burden of antimicrobial resistance. Why it is more serious than current studies suggest. 2013. www.Ishtm.ac.uk/php/economics/assets/dh_amr report.pdf.

9 Coast J, Smith RD, Karcher AM, Wilton P, Millar M. Superbugs II: how should economic evaluation be conducted for interventions which aim to reduce antimicrobial resistance? Health Econ 2002;11:637-7.

10 Gregor M. Bird flu: a virus of our own hatching. New York, 2006:85

11 Cars O, Hogberg LD, Murray M, Jasper W, Nordberg O, Sivaraman S, et al. Meeting the challenge of antibiotic resistance. BMJ 2008;337:726-8.

12 Bratzer DW, Houck PM. Antimicrobial prophylaxis for surgery: an advisory statement from the National Surgical Infection Prevention Project. Clin Infect Dis 2004;38:1706-15.

13 Wild SM. Antibiotic prophylaxis at caesarean section. Lancet 2002;360:724.

14 Plowman RP, Graves N, Griffin M, Roberts JA, Swan AV, Cookson BC, et al. The socioecomic burden of hospital acquired infection. Public Health Laboratory Service, 1999.

15 Kanerva M, Blom M, Tuominen U, Kolho E, Anttila VJ, Vaara M, et al. Costs of an outbreak of methicillin-resistant Staphylococcus aureus. Hosp Infect 2007;66:22-8.

Cite this as: BMJ 2013;346:f1493

(c) BMJ Publishing Group Ltd 2013 


\section{Table}

Table 1 | Annual cost of illness for selected conditions in US

\begin{tabular}{ll} 
Health problem & Societal cost (\$bn, 2004) \\
Cardiovascular disease $^{\mathrm{w} 1}$ & 380 \\
\hline Musculoskeletal conditions $^{\mathrm{w} 2}$ & 300 \\
\hline Motor vehicle accidents $^{\mathrm{w} 3}$ & 270 \\
\hline Occupational injury and illness $^{\mathrm{w} 4}$ & 266 \\
\hline Mental disorders $^{\mathrm{w5}}$ & 260 \\
\hline Substance abuse $^{\mathrm{w} 6}$ & 195 \\
\hline Cancer (all) $^{\mathrm{w} 7}$ & 185 \\
\hline Diabetes $^{\mathrm{w} 8}$ & 145 \\
\hline Alzheimer's disease $^{\mathrm{w} 9}$ & 70 \\
\hline Antimicrobial resistance $^{\mathrm{w10}}$ & 55 \\
\hline Skin disease $^{\mathrm{w11}}$ & 48 \\
\hline Urinary incontinence $^{\mathrm{w} 12}$ & 23 \\
\hline Asthma $^{\mathrm{w13}}$ & 16 \\
\hline
\end{tabular}




\section{Figure}

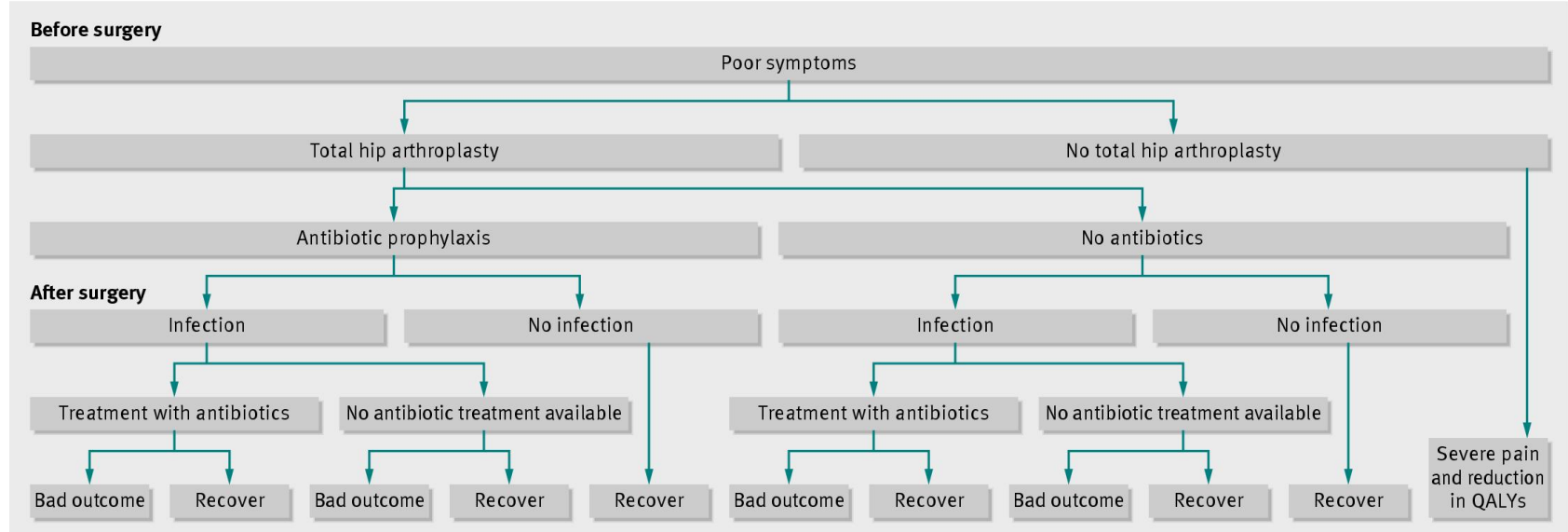

Care pathway for total hip arthroplasty with and without antimicrobials 